\title{
Clinical utility of the platelet function analyzer (PFA-100) in cardiothoracic procedures involving extracorporeal circulation
}

\author{
Sundara Raman, MD, and Norman A. Silverman, MD, Detroit, Mich
}

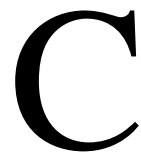

ardiothoracic surgical procedures involving extracorporeal circulation are frequently associated with thrombocytopenia and thromobocytopathy. Although hemodilution and consumption in combination can result in bleeding due to procoagulant factor deficiencies, platelet loss and dysfunction are more commonly responsible for blood loss. Cumbersome test procedures such as bleeding time and platelet aggregation studies do not necessarily correlate with bleeding diatheses. Because of the unavailability of tests with rapid turnaround time to delineate the cause of hemorrhage, various blood components are needlessly used in an attempt to control bleeding. This places a heavy burden on blood banks, especially during times of a nationwide shortage in platelets.

In this communication, we are reporting the results obtained with the platelet function analyzer (PFA-100, Dade Behring, Inc, Deerfield, Ill) on 98 patients with or without hemorrhagic complications after undergoing coronary artery bypass grafting (CABG). The PFA- $100^{1}$ is a high shear system that challenges platelets, in anticoagulated whole blood, to occlude an aperture cut into a membrane coated with collagen and an agonist (epinephrine or adenosine $5^{\prime}$-diphosphate, ADP). Results are expressed in seconds, with a maximum test time of 300 seconds. This system has previously been shown to be sensitive to a variety of platelet and von Willebrand factor defects (inherited, acquired, or drug-induced), as well as hemorheologic factors. ${ }^{1-4}$

\section{Patients and Methods}

Patients were screened preoperatively by prothrombin time, activated partial thromboplastin time, fibrinogen, D-dimer, complete blood count, and platelet count for coagulation abnormalities. Ninety-eight patients undergoing coronary artery bypass for the first time with normal coagulation function and platelet counts and withdrawal of aspirin or other antiplatelet drugs for at least 3 days before the operation were enrolled.

From the Divisions of Hematopathology and Thoracic Surgery, Henry Ford Hospital, Detroit, Mich.

Received for publication Oct 9, 2000; accepted for publication Jan 11, 2001.

Address for reprints: S. Raman, MD, Division of Hematopathology, Henry Ford Hospital, Room WC610, 2799 W Grand Blvd, Detroit, MI 48202 (Email: sraman1@hfhs.org).

J Thorac Cardiovasc Surg 2001;122:190-1

Copyright $(\odot) 2001$ by The American Association for Thoracic Surgery

$0022-5223 / 2001 \$ 35.00+0 \quad \mathbf{1 2 / 5 4 / 1 1 4 3 4 4}$

doi:10.1067/mtc.2001.114344
Surgical procedures were performed with standard cardiopulmonary bypass techniques at moderate systemic hypothermia $\left(30^{\circ} \mathrm{C}-32^{\circ} \mathrm{C}\right)$ with crystalloid pump prime. Heparin sodium was given at an initial dosage of $3 \mathrm{mg} / \mathrm{kg}$, supplemented to maintain the activated clotting time at more than 400 seconds and reversed with protamine sulfate to return activated clotting time to baseline.

Blood samples were collected in $0.109 \mathrm{~mol} / \mathrm{L}$ (3.2\%) trisodium citrate before heparinization and 15 minutes after heparin neutralization by protamine sulfate for measurements of coagulation parameters, complete blood count, and platelets and for PFA-100 measurements. Platelet counts and hematocrit values in the samples obtained after heparin neutralization ranged from $64,000 / \mu \mathrm{L}$ to $202,000 / \mu \mathrm{L}$ and $29.5 \%$ to $38.2 \%$, respectively. Preoperative PFA-100 measurements were within normal limits in all patients with the collagen-ADP cartridge (normal cutoff < 107 seconds).

Patients identified postoperatively as having bleeding abnormalities were initially treated with platelets. Persistent bleeding (such as continuous oozing at incision sites) was treated with fresh frozen plasma when the international normalized ratio was more than 1.6 and with platelets when the platelet count was less than $100,000 / \mu \mathrm{L}$. Laboratory abnormalities alone did not mandate component therapy. Operative re-exploration was undertaken for chest tube output greater than $300 \mathrm{~mL}$ in any hour or greater than 200 $\mathrm{mL}$ per hour for 2 successive hours.

\section{Results and Discussion}

Of the 98 patients, 36 met the criteria for excessive bleeding. For 16 of these patients, bleeding was controlled by platelet transfusion alone. For the other 20, platelet transfusion failed to control the bleeding and other means were necessary. Retrospective analysis of the data showed that PFA-100 measurements performed 15 minutes after administration of protamine were prolonged in 15 of the 16 patients (Table 1) whose bleeding was controlled by platelet transfusion only. This was in sharp contrast to the group without bleeding abnormalities ( 6 of 62 had long PFA-100 measurements) and to the group of patients that required more than platelets to control bleeding, only 3 of 20 having prolonged PFA-100 measurements (Figure 1). Platelet counts alone were inadequate for identifying which patients with bleeding abnormalities would respond to platelet transfusion (Table 1). In contrast, the sensitivity and specificity of the PFA-100 system for identifying response to platelet transfusion were $94 \%$ and $85 \%$, respectively. These observations suggest a potential for PFA-100 measurements to help identify which patients could benefit the most from platelet transfusion if bleeding complications follow a CABG procedure. This observation is in concordance with Slaughter and coworkers, ${ }^{5}$ who reported that $80 \%$ of patients having CABG with abnormal chest tube output had prolonged PFA-100 measurements 15 minutes after administration of protamine. 


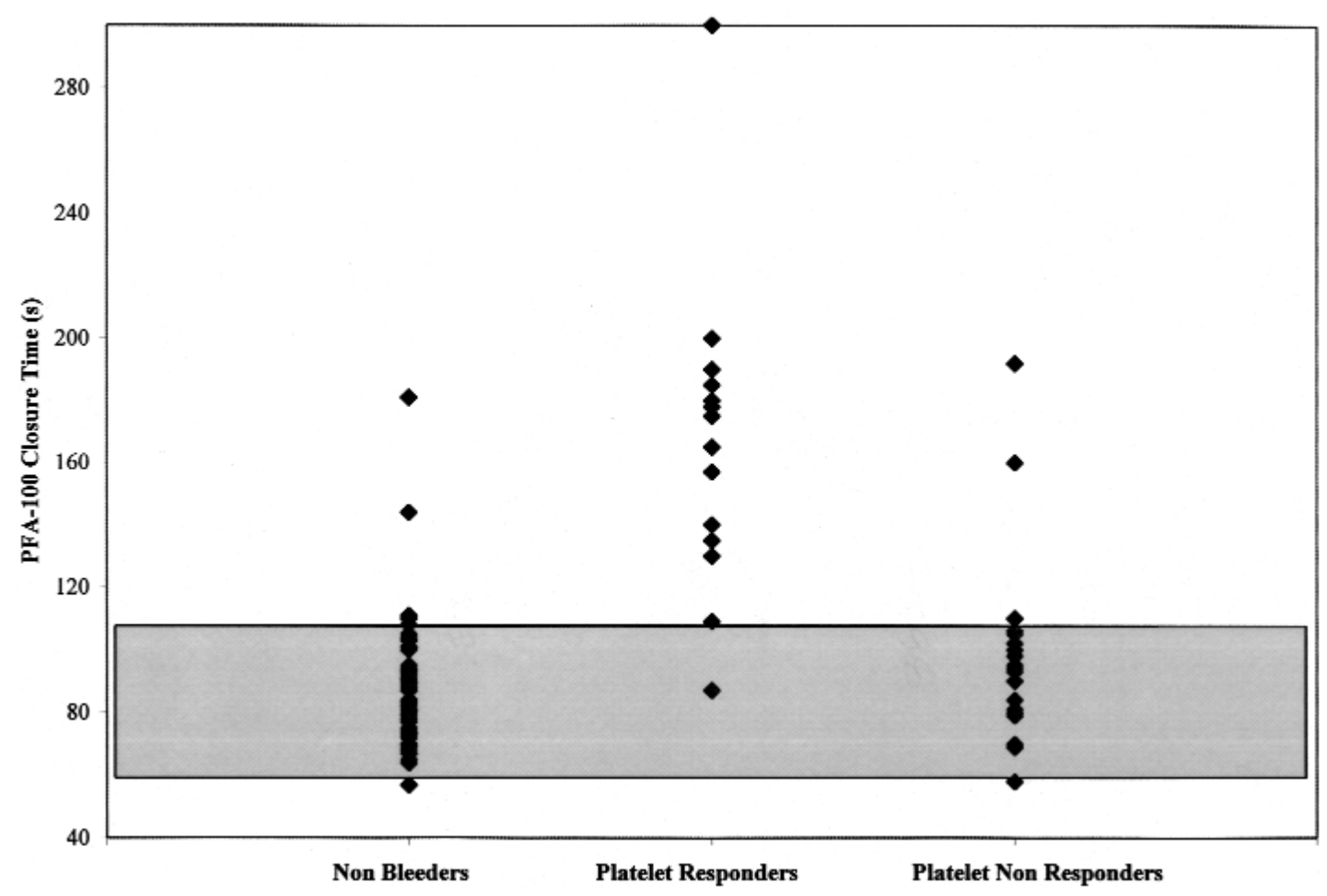

Figure 1. Correlation between closure times and the bleeding classification of 98 patients having CABG: PFA-100 measurements were obtained on $3.2 \%$ citrated blood samples tested with the collagen-ADP cartridge. Similar results were obtained with the collagen-epinephrine cartridge (results not shown). The shaded box indicates the normal range for collagen-ADP determined on 105 ostensibly normal donors (59-107 seconds).

TABLE 1. Sensitivity and specificity of platelet counts and PFA-100 measurements

\begin{tabular}{lcl}
\hline Type of bleeding abnormality & Platelet count & PFA-100 \\
\hline PR $(n=16)$ & $56 \%$ sensitivity $(9 / 16)$ & $94 \%$ sensitivity $(15 / 16)$ \\
PNR $(n=20)$ & $55 \%$ specificity $(11 / 20)$ & $85 \%$ specificity $(17 / 20)$ \\
\hline
\end{tabular}

Sensitivity and specificity of platelet counts and PFA-100 measurements (collagen-ADP cartridge) for identifying patients with bleeding abnormalities that were responsive to platelets $(P R)$ and those that were not (PNR). Platelet counts were considered abnormal if less than 100,000/ $\mu \mathrm{L}$. PFA-100 results were considered abnormal if the aperture occlusion time was more than 107 seconds.

\section{Conclusions}

Our study involving a small number of patients suggests that use of the PFA-100 system to monitor platelet functional status is beneficial in cardiothoracic surgical procedures involving extracorporeal circulation. Specifically, had the PFA-100 system been used to direct postoperative transfusion in patients with bleeding problems, $50 \%$ of these patients would have been spared platelets with an accuracy of $94 \%$ (17/18). The simplicity of the procedure, which enables the test to be performed on-site, the lack of significant preanalytical variables, and the rapid turnaround time make this instrument an ideal tool for this purpose.

\section{References}

1. Mammen EF, Comp PC, Gosselin R, Greenberg C, Hoots WK, Kessler CM, et al. PFA-100 system: a new method for assessment of platelet dysfunction. Semin Thromb Hemost. 1998;24:195-202.

2. Hezard N, Metz D, Nazeyrollas P, Droulle C, Elaerts J, Potron G, et al. Use of the PFA-100 apparatus to assess platelet function in patients undergoing PTCA during and after infusion of c7E3 Fab in the presence of other antiplatelet agents. Thromb Haemost. 2000;3:540-4.

3. Homoncik M, Jilma B, Hergovich N, Stohlawetz P, Panzer S, Speiser W. Monitoring of aspirin pharmacodynamics with the platelet function analyzer PFA-100. Thromb Haemost. 2000;83:316-21.

4. Raman S, Shurafa M, Mercado R, Cantwell L, Canoso A, Wong W. Comparison of bleeding time, platelet aggregation and platelet function using PFA-100 in different groups of patients with relevant clinical and laboratory correlations [abstract]. Thromb Haemost. 1999;82:742.

5. Slaughter T, Sreeram G, Sharma A, Greenberg C. Shear-mediated platelet dysfunction during cardiac surgery: mechanism and clinical implications [abstract]. Thromb Haemost. 1999;82:744. 